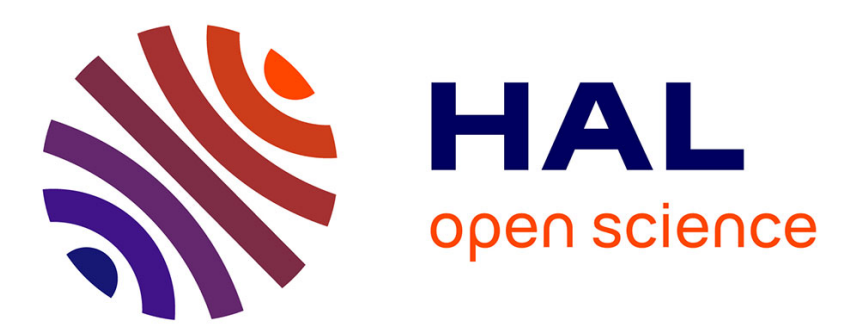

\title{
Modifications des caractéristiques électriques de contacts métal-silicium par bombardement d'ions argon de faible énergie
}

\author{
A. Chouiyakh, B. Lang
}

\section{- To cite this version:}

A. Chouiyakh, B. Lang. Modifications des caractéristiques électriques de contacts métal-silicium par bombardement d'ions argon de faible énergie. Revue de Physique Appliquée, 1984, 19 (12), pp.971-978. 10.1051/rphysap:019840019012097100 . jpa-00245293

\section{HAL Id: jpa-00245293 https://hal.science/jpa-00245293}

Submitted on 1 Jan 1984

HAL is a multi-disciplinary open access archive for the deposit and dissemination of scientific research documents, whether they are published or not. The documents may come from teaching and research institutions in France or abroad, or from public or private research centers.
L'archive ouverte pluridisciplinaire HAL, est destinée au dépôt et à la diffusion de documents scientifiques de niveau recherche, publiés ou non, émanant des établissements d'enseignement et de recherche français ou étrangers, des laboratoires publics ou privés. 


\title{
Modifications des caractéristiques électriques de contacts métal-silicium par bombardement d'ions argon de faible énergie
}

\author{
A. Chouiyakh et B. Lang \\ Laboratoire de Cristallographie, ERA no 7 du CNRS et Université Louis Pasteur, \\ 4, rue Blaise Pascal, 67000 Strasbourg, France
}

(Reçu le 6 janvier 1984, révisé le 29 mai, accepté le 13 septembre 1984)

\begin{abstract}
Résumé. - Les caractéristiques $I(V)$ d'un contact métal-Si monocristallin faiblement dopé sont radicalement modifiées par bombardement d'ions argon : le contact métal-Si(n) devient ohmique, le contact métal-Si(p) devient redresseur. L'effet d'irradiation est considérable même pour des énergies de l'ordre de $100 \mathrm{eV}$. La couche de surface amorphe, enrichie en donneurs, donne naissance à des structures métal- $n^{+}-n$ et métal $-n^{+}-p$. La conduction est dominée par l'interface Si(amorphe)-Si(cristal) dans la profondeur du matériau. Les caractéristiques électriques sont comparées à celles de jonctions Si(amorphe)-Si(cristal) ou Si(endommagé)-Si(cristal) réalisées par d'autres techniques.
\end{abstract}

\begin{abstract}
Argon ion bombardment changes completely the nature of metal-Si contacts : the metal-Si(n) contact becomes ohmic, the metal-Si(p) contact becomes rectifying. The radiation effects are large even for energies as low as $100 \mathrm{eV}$. The amorphous surface layer is enriched in donors, thus giving rise to metal $-n^{+}-n$ and metal $-n^{+}-p$ structures. The conduction process is dominated by the amorphous-crystalline interface in the depth of the $\mathrm{Si}$ substrate. Comparison is made with amorphous-crystalline or damaged-crystalline junctions made by other techniques.
\end{abstract}

\section{Introduction.}

Le bombardement par des ions argon (de quelques centaines d'eV à quelques $\mathrm{keV}$ ), suivi d'un recuit, est une méthode courante pour la préparation de surfaces monocristallines atomiquement propres. Nous l'avons appliqué au silicium en combinaison avec la spectroscopie des électrons Auger (SEA) en vue de caractériser les surfaces propres $\mathrm{Si}(111)$ et (100) et d'étudier leur oxydation [1-4].

Plus récemment, nous avons étudié l'amorphisation d'une surface de Si par bombardement ionique et son évolution lors du recuit thermique [5].

A partir de ces bases, nous avons recherché l'influence des traitements de la surface du Si (décapage ionique, traitement thermique, oxydation) sur les propriétés électriques du contact métal-Si. Notre approche est donc celle de physico-chimistes des surfaces cherchant à corréler des propriétés à l'échelle atomique (structure, composition, ...) et des propriétés macroscopiques (caractéristiques courant-tension).

En celà, nous nous distinguons des spécialistes des dispositifs à semiconducteurs qui, habitués aux technologies utilisant les faisceaux d'ions (sputter-deposition, sputter-etching), cherchent à remonter aux modifications de la surface à l'échelle atomique [6] ou bien qui étudient le mécanisme de formation de la barrière à la surface du Si traité par décapage ionique [7].

\section{Préparation et caractérisation.}

Une expérience typique se déroule en trois étapes :

a) bombardement ionique dans une enceinte à ultra-vide [2] ;

b) métallisation dans une enceinte à vide classique ;

c) mesure de la caractéristique $I(V)$ à l'air.

Ce mode opératoire ne nous permet pas d'accéder aux propriétés du contact intime métal-Si. On peut envisager de réaliser les trois étapes in situ comme l'ont fait Testemale et al. [7] pour quelques expériences ponctuelles. Cepc idant, le nombre de paramètres (énergie, dose, incidence, nature des ions de gaz rare; type de dopage, résistivité, face du cristal ; nature du métal) ainsi que la nécessité de changer de plaquette après chaque expérience, de manière à éliminer le facteur " histoire antérieure " de l'échantillon, rendraient une étude tant soit peu détaillée prohibitivement longue. De plus, il n'est pas facile d'assurer, sous vide, de bons contacts avec le circuit externe de mesures électriques. 
Nous prouverons plus loin que notre mode opératoire n'introduit que des différences minimes par rapport à une expérience faite in situ. Il trouvera sa justification a posteriori dans la discussion qui établira que l'interface métal-Si n'a qu'une importance relative; c'est l'interface $\mathrm{Si}$ amorphisé-Si cristallin (dans la profondeur de l'échantillon) qui joue le rôle déterminant.

Dans ces conditions, la brève remise à l'air lors du transfert de l'enceinte à ultra-vide dans la cloche d'évaporation n'est pas gênante. Nous avons d'ailleurs contrôlé par SEA l'état d'oxydation de la surface bombardée suivant la durée d'exposition à l'air.

En nous basant sur la structure fine du spectre Auger $\mathrm{SiL}_{23} \mathrm{VV}[3,4]$, nous trouvons :

a) pour des durées de 20-100 min, une couche oxydée de $7-9 \AA$ correspondant à l'oxyde intermédiaire non stoechiométrique, plus une faible quantité de "quasi-silice " $\left(\mathrm{SiO}_{2}\right.$ en couche mince différente de la silice massive);

b) pour une exposition à l'air de 1 à 3 jours, une couche oxydée de $14-15 \AA$ correspondant à l'oxyde intermédiaire plus une couche complète de "quasisilice ".

L'oxydation à l'air conduit à une saturation assez rapide, puisque les plaquettes brutes de $\mathrm{Si}$ ont une épaisseur d'oxyde d'environ $17 \AA$; à ce stade, la silice "massive " a commencé à croître.

En résumé, nos conditions opératoires (durée du transfert sous pression atmosphérique : environ $15 \mathrm{~min}$ ) sont telles qu'une mince couche d'oxyde $(<10 \AA)$ s'interpose entre le Si et le dépôt métallique. Nos échantillons sont découpés dans des plaquettes d'épaisseur $300 \mu$ : $\operatorname{Si}(111)-n \quad(5,4-6,2 \Omega \times \mathrm{cm})$ et $\mathrm{Si}(100)-\mathrm{p}(8,5-11,5 \Omega \times \mathrm{cm})$.

La face présentant un poli spéculaire est soumise au décapage ionique; l'autre face, rodée, recevra le contact ohmique. Avant montage l'échantillon est attaqué 2 min dans HF concentré.

Les conditions de bombardement ionique sont : ions argon de $80 \mathrm{eV}$ à $3 \mathrm{keV}$, densité de courant de quelques $\mu \mathrm{A} / \mathrm{cm}^{2}$, dose d'irradiation : $3 \times 10^{16}$ ions/ $\mathrm{cm}^{2}$, incidence normale, température ambiante. Un porte-échantillon du type «carrousel » permet de traiter quatre échantillons à tour de rôle sans rompre le vide.

Le bombardement ionique se fait dans les conditions les plus propres possibles : enceinte à ultra-vide étuvée; pompe ionique au titane isolée par une vannetiroir pour éviter qu'elle ne régurgite des gaz contaminants; sublimateur de $\mathrm{Ti}$ en marche à la fois pour purifier l'argon et pour minimiser la remontée de pression des gaz résiduels. Le spectre Auger montre que la surface ainsi bombardée est exempte d'oxygène et contient très peu de carbone (rapport du pic Auger du $\mathrm{C}$ à celui du $\mathrm{Si}$ : $0,3 \%$ ).

Bien que l'échantillon bombardé se contamine superficiellement lors du transfert, il est bon de soigner la propreté du bombardement, de manière à ne pas contaminer les couches sous-jacentes par implantation d'impuretés qui pourraient migrer par diffusion assistée sous irradiation et former, par exemple, des complexes impuretés-lacunes; d'autre part, on cherche à éviter les impuretés (autres que l'argon) pouvant influer sur la recristallisation ultérieure [8].

La métallisation est faite d'abord sur la face bombardée $(\mathrm{Au}, \mathrm{Ag})$, puis sur la face arrière (contact ohmique : Al sur Si-n, Au sur Si-p). L'épaisseur du dépôt est comprise entre 500 et $1000 \AA$; elle n'est critique que pour $\mathrm{Al}$ : pour des épaisseurs inférieures à $1000 \AA$, on note une évolution rapide des caractéristiques en fonction du temps. Lé dépôt sur la face avant a un diamètre de $2 \mathrm{~mm}$; le dépôt sur la face arrière est rectangulaire $9 \times 3 \mathrm{~mm}$.

L'aire bombardée est $6 \times 10 \mathrm{~mm}$; son homogénéité a été contrôlée en déposant le métal en différents endroits.

Les courbes $I(V)$ sont mesurées à la température ambiante; elles sont enregistrées directement sur table traçante. Le contact avec le circuit externe de mesure est réalisé par deux billes d'or $(\varnothing 1,5 \mathrm{~mm})$ brasées sur deux lames faisant ressort et appliquant une légère pression sur les électrodes métalliques.

$\mathrm{Vu}$ le dopage utilisé $\left(\sim 10^{15} \mathrm{~cm}^{-3}\right)$, il est difficile d'obtenir un bon contact ohmique "face arrière"; d'autant plus que la barrière d'un contact $\mathrm{Al}-\mathrm{Si}$ fraîchement déposé est sensible aux conditions de dépôt et qu'il peut y avoir vieillissement à l'air : le contact $\mathrm{Al}-\mathrm{Si}(\mathrm{n})$ tend à devenir redresseur. De suite après dépôt et sans recuit, sa résistance est de 2-15 $\Omega$. Cette incertitude se répercute sur les valeurs des résistances de contact mesurées $(\S 3)$ que nous donnons avec une certaine "fourchette".

Il est donc essentiel d'avoir des échantillons de référence, non traités, métallisés dans les mêmes conditions que ceux bombardés. Ils possèdent les caractéristiques attendues : type "diode" pour $\mathrm{Au} / \mathrm{Si}-\mathrm{n} / \mathrm{Al}$ avec une résistance de $2 \times 10^{4} \Omega$ et une barrière de $0,72 \mathrm{~V}$; ohmique pour $\mathrm{Au} / \mathrm{Si}-\mathrm{p} / \mathrm{Au}$ avec une résistance de $4 \Omega$.

La différence entre échantillons bombardés et références est telle (cf. § 3) que la plus ou moins bonne qualité du contact "face arrière " ne change pas la réalité physique des phénomènes décrits.

\section{Résultats.}

La surface de référence est attaquée par HF, rincée à l'eau et conservée sous méthanol jusqu'au montage dans la cloche d'évaporation. D'après la SEA, l'épaisseur oxydée ne dépasse pas 4-5 $\AA$.

3.1 CAS DU Si-n. - Au-dessus de $900 \mathrm{eV}$, les courbes $I(V)$ sont franchement ohmiques (Fig. 1) et ne se modifient pratiquement pas jusqu'à $3 \mathrm{keV}$.

En polarisation directe, le courant est toujours fort quelle que soit l'énergie entre $80 \mathrm{eV}$ et $3 \mathrm{keV}$. La résistance différentielle : $R_{0}=(\mathrm{d} V / \mathrm{d} I)_{V \rightarrow 0}$ (inverse 


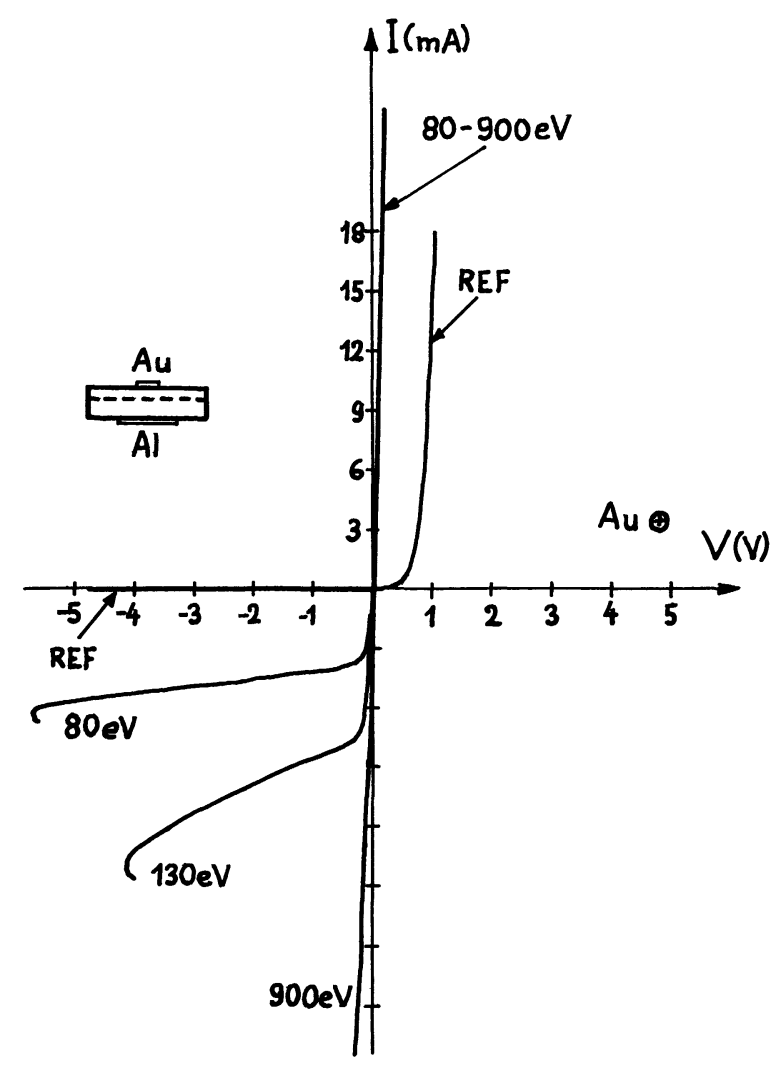

Fig. 1. - Caractéristiques $I(V)$ de structures $\mathrm{Au} / \mathrm{Si}-\mathrm{n} / \mathrm{Al}$ en fonction de l'énergie des ions argon; la courbe REF correspond à une attaque chimique par HF.

$[I(V)$ curves of $\mathrm{Au} / \mathrm{Si}-\mathrm{n} / \mathrm{Al}$ structures as a function of argon ion energy ; the REF curve corresponds to a HF etch.]

de la pente à l'origine) est faible et varie peu en fonction de l'énergie des ions : $R_{0}=6-20 \Omega$. Elle est du même ordre que la résistance du volume : $R_{\mathrm{v}}=$ $10 \Omega$. A partir de $R_{0}$, on peut estimer une résistance de contact $R_{\mathrm{C}}$ (définie comme le produit de $R_{0}$ par l'aire du contact) de $0,5 \Omega \times \mathrm{cm}^{2}$.

En polarisation inverse, la caractéristique est beaucoup plus sensible à l'énergie des ions. A mesure que l'énergie diminue, le courant inverse diminue et tend vers une saturation.

Aux faibles énergies, on tend donc vers un contact médiocrement redresseur. Si l'on applique les équations d'une barrière de Schottky, on peut évaluer, à partir des courbes $\log I=f(V)$ (Fig. 2) un facteur d'idéalité $n$ et une hauteur de barrière $\Phi_{\mathrm{B}}$. Pour l'énergie la plus faible $(80 \mathrm{eV})$, on trouve : $n=2,9$ et $\Phi_{\mathrm{B}}=0,47 \mathrm{~V}$.

Les caractéristiques obtenues sont semblables pour $\mathrm{Au}$ et $\mathrm{Ag}$.

3. 2 CAS DU Si-p. - Les courbes $I(V)$ (Fig. 3) sont typiques d'un contact redresseur quelle que soit l'énergie des ions dans la gamme $80 \mathrm{eV}$ à $3 \mathrm{keV}$. Les caractéristiques semi-log (Fig. 4) sont linéaires sur 4 modules.

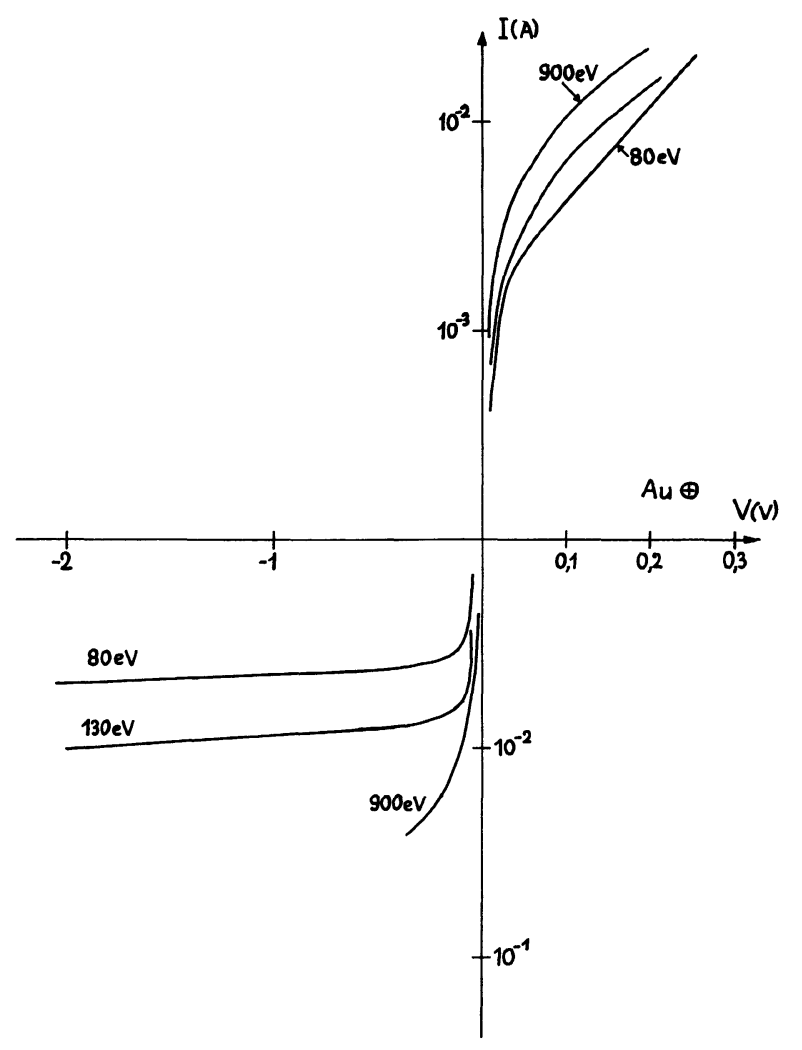

Fig. 2. - Caractéristiques $\log (I)=f(V)$ dans les mêmes conditions que la figure 1.

[ $\log I=f(V)$ curves in the same conditions as figure 1.]

Elles tendent à s'améliorer à mesure que l'énergie des ions augmente, comme le montre le tableau I. Toutefois, les variations des paramètres du contact ne sont appréciables qu'aux plus faibles énergies de bombardement, entre 80 et $250 \mathrm{eV}$ (Fig. 5). La résistance-série est comparable à la résistance du volume de l'échantillon.

On notera que le caractère redresseur est indépendant de la nature du métal : les courbes $I(V)$ sont semblables pour $\mathrm{Au}, \mathrm{Ag}$ ou $\mathrm{Al}$.

3. 3 COMPARAISON AVEC LES CONTACTS FABRIQUÉS in situ. - Nos résultats sont extrêmement voisins de ceux obtenus par Testemale et al. [7] qui font la métallisation in situ. A dopage et énergie des ions identiques $(3 \mathrm{keV})$, nous trouvons la même densité de courant de saturation $\left(J_{S}=8 \times 10^{-6} \mathrm{~A} / \mathrm{cm}^{2}\right)$ pour le contact $\mathrm{Ag} / \mathrm{Si}(100)$-p et une résistance de contact comparable $R_{\mathrm{C}}=0,6 \Omega \times \mathrm{cm}^{2}$ pour $\mathrm{Ag} / \mathrm{Si}(111)-\mathrm{n}$ au lieu de $0,25 \Omega \times \mathrm{cm}^{2}$ pour $\mathrm{Ag} / \mathrm{Si}(100)-\mathrm{n}$; l'écart est du même ordre que le rapport calculé [9] : $R_{\mathrm{C}}(100) /$ $R_{\mathrm{C}}(111) \simeq 0,5$.

\section{Discussion.}

Après avoir rappelé les principaux résultats, nous essayerons de les interpréter en termes de modèles connus de jonctions. Nous soulignerons l'importance 


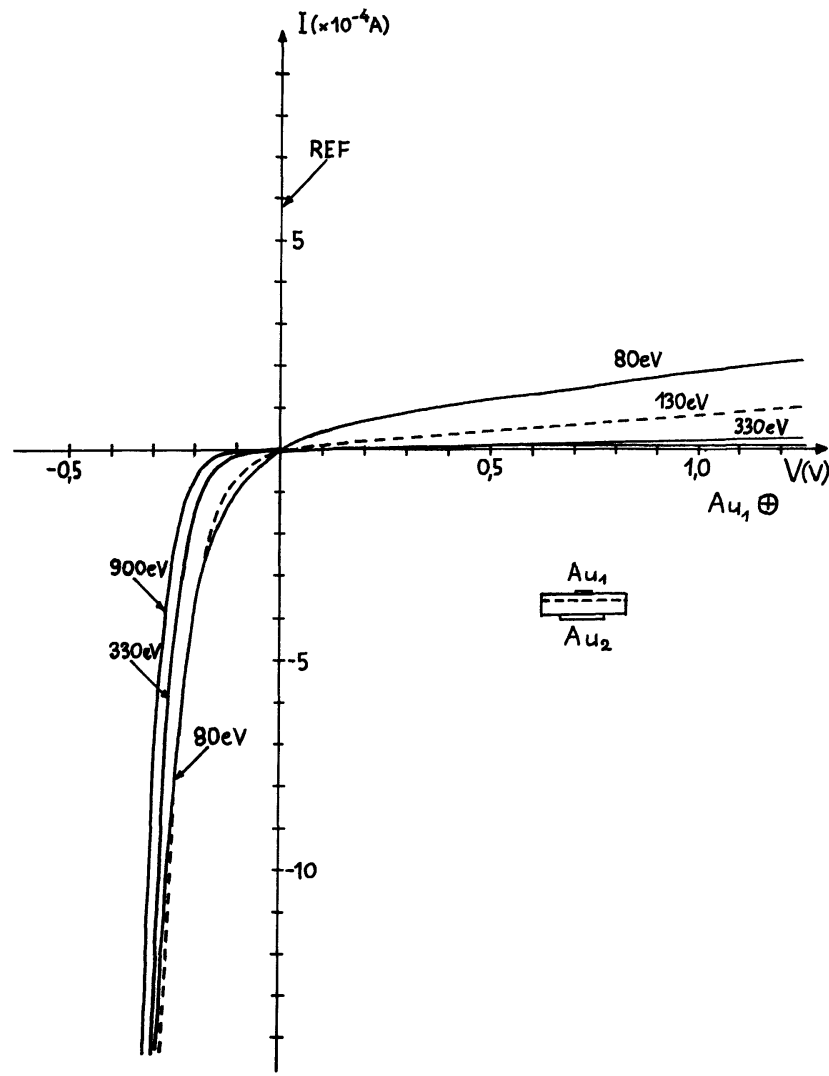

Fig. 3. - Caractéristiques $I(V)$ de structures $\mathrm{Au} / \mathrm{Si}-\mathrm{p} / \mathrm{Au}$ en fonction de l'énergie des ions argon; la courbe REF correspond à une attaque chimique par HF : elle se confond pratiquement avec l'axe des $I$.

$[I(V)$ curves of $\mathrm{Au} / \mathrm{Si}-\mathrm{p} / \mathrm{Au}$ structures as a function of argon ion energy; the REF curve, corresponding to a HF etch, and the $I$ axis practically merge together.]

de la couche interfaciale créé par le bombardement ionique. Enfin, nous établirons une comparaison avec d'autres traitements de surface réalisant la transition d'une couche superficielle perturbée au substrat monocristallin.

Les principaux résultats peuvent être récapitulés de la manière suivante :

- le bombardement d'ions argon rend ohmique un contact métal/Si-n qui est normalement redres-

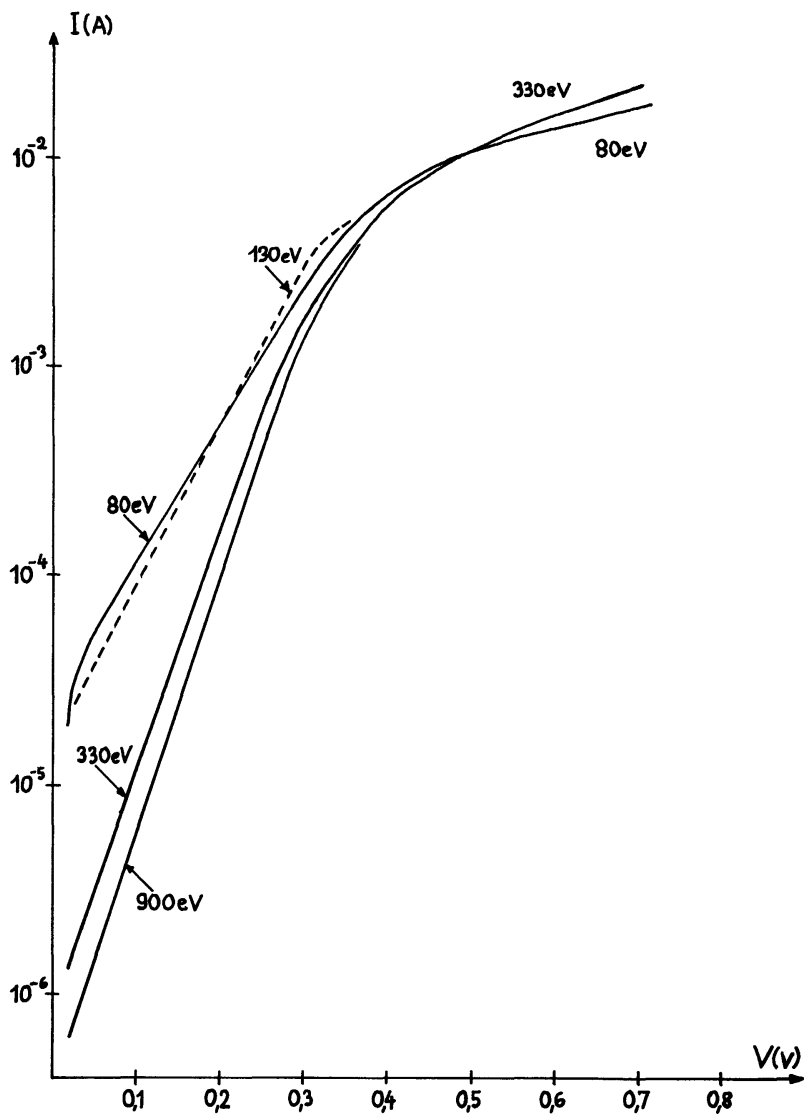

Fig. 4. - Caractéristiques $\log (I)=f(V)$ dans les mêmes conditions que la figure 3.

$[\log I=f(V)$ curves in the same conditions as figure 3.]

seur; il rend redresseur un contact métal/Si-p qui est normalement ohmique;

- des ions d'énergie aussi faible que $80 \mathrm{eV}$ modifient radicalement la nature du contact (davantage pour le type $p$ que pour le type $n$ );

- les caractéristiques n'évoluent pratiquement plus (pour les deux types) lorsque l'énergie des ions dépasse $1 \mathrm{keV}$;

- la nature du contact dépend peu du métal utilisé (cf. tableau II).

Tableau I. - Evolution des paramètres électriques de structures $\mathrm{Au} / \mathrm{Si}-\mathrm{p} / \mathrm{Au}$ en fonction de l'énergie des ions argon.

[Electrical parameters of $\mathrm{Au} / \mathrm{Si}-\mathrm{p} / \mathrm{Au}$ structures as a function of argon ion energy.]

\begin{tabular}{lccccccc}
\hline$E_{\text {ion }}(\mathrm{eV})$ & 80 & 130 & 250 & 330 & 600 & 730 & 900 \\
\hline$\Phi_{\mathrm{B}}(\mathrm{V})$ & 0,65 & 0,66 & 0,70 & 0,71 & 0,72 & 0,72 & 0,73 \\
$n$ & 2,52 & 2,15 & 1,75 & 1,53 & 1,48 & 1,40 & 1,38 \\
$R_{0}\left(\times 10^{5} \Omega\right)$ & 0,012 & 0,033 & 0,32 & 0,50 & $\simeq 1$ & $>1$ & $>1$ \\
\hline
\end{tabular}


Tableau II. - Comparaison d'interfaces $\mathrm{Si}($ amorphe)-Si(cristal) préparés par différentes techniques.

[Comparison of amorphous Si-crystalline $\mathrm{Si}$ interfaces prepared by various techniques.]

\begin{tabular}{|c|c|c|c|c|}
\hline $\begin{array}{l}\text { Technique de préparation } \\
\text { de } \operatorname{Si}(a)\end{array}$ & $\begin{array}{l}\text { Epaisseur } \\
\text { de Si(a) }\end{array}$ & $\begin{array}{l}\text { Traitement } \\
\text { du substrat }\end{array}$ & $\begin{array}{c}\text { Contact } \\
\text { avec } \operatorname{Si}(a)\end{array}$ & Réf. \\
\hline $\begin{array}{l}\text { Bombardement par ions } \\
\text { argon } \\
i d . \\
i d .\end{array}$ & $\begin{array}{l}20-100 \AA \\
\quad \text { id. } \\
\quad \text { id. }\end{array}$ & $\begin{array}{l}\text { attaque } \\
\text { ionique } \\
\quad \text { id. } \\
\text { id. }\end{array}$ & $\begin{array}{l}\mathrm{Au}, \mathrm{Ag}, \mathrm{Al} \\
\mathrm{Au}, \mathrm{Ti}, \mathrm{Al} \\
\mathrm{Ag}\end{array}$ & $\begin{array}{c}\text { présent } \\
\text { travail } \\
{[6]} \\
{[7]}\end{array}$ \\
\hline Evaporation & $100 \AA$ & $\begin{array}{l}\text { attaque } \\
\text { chimique }\end{array}$ & $?$ & [19] \\
\hline $\begin{array}{l}\text { Pulvérisation cathodique } \\
\text { (sputtering) }\end{array}$ & $600 \AA$ & $\begin{array}{l}\text { attaque } \\
\text { ionique }\end{array}$ & $\mathrm{Au}$ & [20] \\
\hline$i d$ & $3000 \AA$ & $\begin{array}{l}\text { attaque } \\
\text { chimique }\end{array}$ & $\mathrm{Sb}$ & [21] \\
\hline id. & $1000 \AA$ & $\begin{array}{l}\text { attaque } \\
\text { ionique }\end{array}$ & $\mathrm{Ag}$ & [22] \\
\hline
\end{tabular}

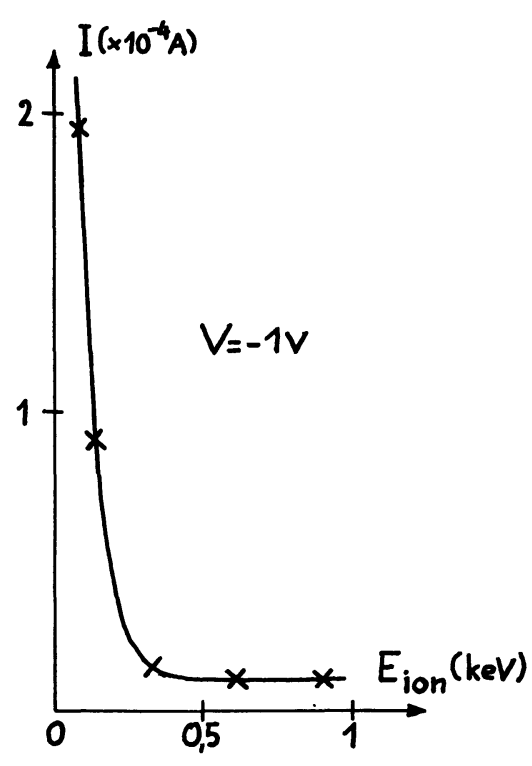

Fig. 5. - Variation du courant inverse en fonction de l'énergie des ions à polarisation fixe : $-1 \mathrm{~V}$; structure $\mathrm{Au} / \mathrm{Si}-\mathrm{p} / \mathrm{Au}$.

$\lceil\mathrm{Plot}$ of reverse current as a function of ion energy at fixed bias (-1 V); Au/Si-p/Au structure.]

Examinons d'abord les contacts obtenus par modification du Si-n. Aux fortes énergies ( $>1 \mathrm{keV})$, la barrière est détruite par le bombardement. Aux énergies plus faibles, la caractéristique devient dissymétrique : il existe donc une barrière, mais sa résistance est fortement diminuée.

$\mathrm{La}$ résistance différentielle est du même ordre que la résistance du volume : $R_{0} \sim R_{\mathrm{v}}$; on a donc affaire à un contact hybride qu'il est difficile de classer dans une catégorie bien définie : $\mathrm{ni}$ barrière de Schottky $\left(R_{0} \gg R_{\mathrm{V}}\right)$, ni ohmique $\left(R_{0} \ll R_{\mathrm{V}}\right)$.

On connait, aussi bien par le calcul que par l'expérience, la variation de la résistance de contact du Si-n en fonction du dopage $[9,10]$. Une valeur $R_{\mathrm{C}}=$ $3-6 \times 10^{-1} \Omega \times \mathrm{cm}^{2}$, associée à une barrière de $0,4-$ $0,5 \mathrm{~V}$, correspondrait à une concentration en donneurs (supposée uniforme) de la couche superficielle comprise entre $5 \times 10^{17}$ et $10^{18} \mathrm{~cm}^{-3}$. Une telle valeur situe les contacts étudiés dans une zone de transition entre l'émission thermoïonique pure par-dessus la barrière et l'effet tunnel à travers la barrière [10].

Le bombardement ionique enrichit donc la surface en centres donneurs (couche d'accumulation) donnant naissance à l'équivalent d'une jonction $\mathrm{n}^{+}-\mathrm{n}$. Une telle jonction ne possède pas de zone interfaciale à forte résistance.

Le contact métal-Si-n bombardé peut être envisagé comme une structure combinant une couche de déplétion, correspondant à l'interface métal- ${ }^{+}$, et une couche d'accumulation due à une homojonction isotype $\mathrm{n}^{+}-\mathrm{n}$ (ou hétérojonction, si l'on admet que le Si amorphisé par les ions est un matériau différent du Si cristallin).

Le fonctionnement d'une telle structure dépend de l'importance relative des deux couches, donc de l'énergie des ions. Plus précisément, il faut considérer l'épaisseur de la couche amorphisée $X_{\mathrm{n}}+$ par rapport à la largeur de la barrière métal- $\mathrm{n}^{+}$déduite du dopage $N_{\mathrm{D}^{+}}$de la couche superficielle du Si.

On peut essayer de représenter le contact métal-n ${ }^{+}$ (Fig. 6a), la jonction $n^{+}-n$ (Fig. 6b) et leur interaction (Fig. 6c); on admet que $N_{\mathrm{D}^{+}}$et $\Phi_{\mathrm{B}}$ sont indépendants de l'énergie des ions, respectivement : $10^{18} \mathrm{~cm}^{-3}$ et $0,45 \mathrm{~V}$. On connaît : $N_{\mathrm{D}}=10^{15} \mathrm{~cm}^{-3}$ et $E_{\mathrm{C}}(\mathrm{n})=0,3 \mathrm{~V}$. 


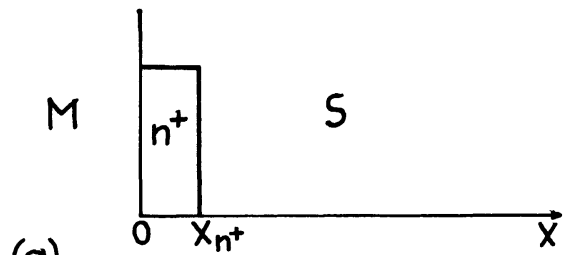

(a)
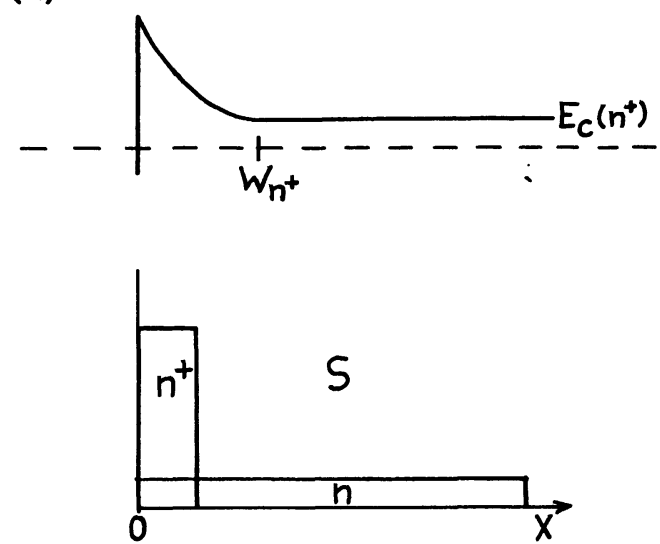

(b)
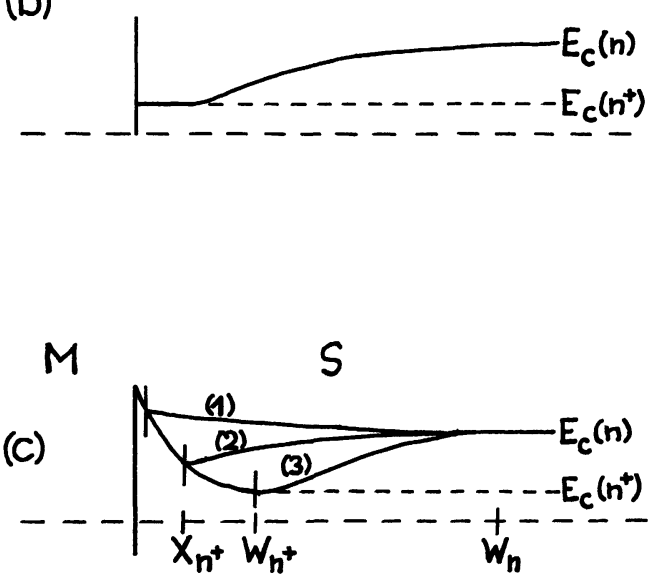

Fig. 6. - Diagrammes de bandes de structures : a) métal$\mathrm{n}^{+}$; b) $\mathrm{n}^{+}-\mathrm{n}$; c) métal- $\mathrm{n}^{+}-\mathrm{n}$; dans le diagramme c), les courbes 1,2 et 3 correspondent à des épaisseurs croissantes de la couche $\mathrm{n}^{+}$.

[Band diagrams for : a) metal- $n^{+}$, b) $n^{+}-n$, c) metal- $n^{+}$. $\mathrm{n}$ structures ; in c) curves 1), 2) and 3) correspond to increasing widths of the $\mathrm{n}^{+}$layer.]

On calcule donc pour le contact métal- $n^{+}: W_{n^{+}}=$ $200 \AA$ et pour la jonction $\mathrm{n}^{+}-\mathrm{n}: E_{\mathrm{C}}(\mathrm{n})-E_{\mathrm{C}}\left(\mathrm{n}^{+}\right)=$ $0,18 \mathrm{~V}$. Les deux zones ne sont séparées que si $X_{n^{+}}>$ $W_{\mathrm{n}^{+}}$; on rejoint alors le modèle de contact ohmique proposé par Popovic [11]. Lorsque $X_{\mathrm{n}^{+}}<W_{\mathrm{n}^{+}}$, la couche dépeuplée s'étale au-delà de la couche amorphisée et $E_{\mathrm{C}}(X)$ présente deux branches à gradients différents. On notera (Fig. 6c) qu'au-delà d'une certaine épaisseur critique la zone de charge d'espace

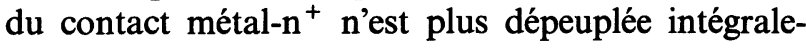
ment.

D'autre part, lorsque l'interface métal- $\mathrm{n}^{+}$est en polarisation directe, la jonction $\mathrm{n}^{+}-\mathrm{n}$ est en polari- sation inverse et vice versa. On conçoit qu'aux faibles polarisations directe (métal +) et inverse (métal -), la couche d'accumulation contrôle la conduction. Aux fortes polarisations directes l'effet d'accumulation domine, tandis qu'aux fortes polarisations inverses, la couche de déplétion absorbe la couche d'accumulation. Suivant l'énergie des ions et suivant la valeur de la polarisation, l'un ou l'autre des interfaces peut contrôler le fonctionnement de la structure métal- $n^{+}-n$.

Dans le cas de contacts obtenus par modification du Si-p, le redressement observé est attribué à une couche d'inversion donnant naissance à une jonction n-p. La structure est composée de deux interfaces : métal- $n^{+}$et $n^{+}-p$; leur interaction est schématisée dans la figure 7. Notons que l'existence d'une couche $n$ à la surface du Si-p bombardé par des ions argon a été détectée dès les années 1960 par Law [12] et Heiland [13].

Si l'on dépose $\mathrm{Al}$ sur Si-p, le contact de référence est déjà redresseur au départ; le bombardement ionique transforme la diode en une jonction n-p. Un cas similaire où l'on implante une diode Al-Si-p avec des donneurs a été analysé par Pai [14].

Examinons la nature de la couche interfaciale créée par le bombardement ionique. La dose d'irradiation utilisée $\left(3 \times 10^{16}\right.$ ions $\left./ \mathrm{cm}^{2}\right)$ est supérieure à celle correspondant à la saturation des dégâts : $5 \times 10^{15}$ ions $/ \mathrm{cm}^{2}$ $[15,16]$. Il en résulte une couche amorphe sur une profondeur de 10-100 $\AA$ suivant l'énergie des ions. On peut estimer l'épaisseur de la couche fortement endommagée à. : $R_{\mathrm{p}}+2 \sigma_{\mathrm{p}}$, où $R_{\mathrm{p}}$ est le parcours projeté et $\sigma_{\mathrm{p}}$ la dispersion correspondante; elle varie de $12 \AA$ pour $100 \mathrm{eV}$ à $120 \AA$ pour $3 \mathrm{keV}$. Nous assimilons la zone la plus endommagée du point de vue structural à la couche électrique enrichie en donneurs : $X_{\mathrm{n}}+\simeq R_{\mathrm{p}}+$ $2 \sigma_{\mathrm{p}}$. C'est une approximation grossière, car au-delà de la zone amorphe, il y a raccordement progressif au monocristal intact au travers d'une zone de transition de moins en moins perturbée. Des tests de dissolution combinés avec des mesures $I(V)$ montrent qu'il existe des défauts électriques à des profondeurs 100 fois plus grandes que la pénétration des ions argon. Les tests $C(V)$ indiquent aussi une jonction graduelle.

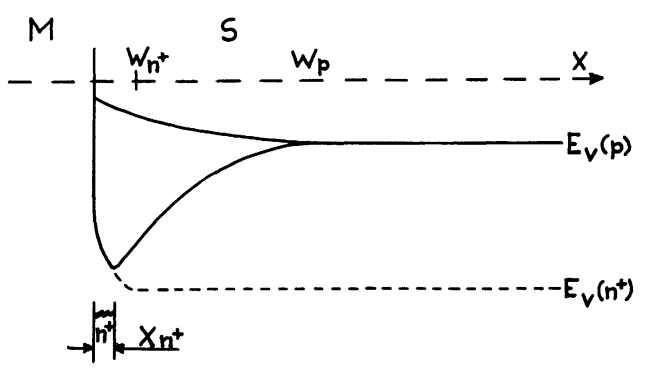

Fig. 7. - Diagramme de bandes d'une structure : métal$\mathrm{n}^{+}-\mathrm{p}$.

[Band diagram of a metal- $n^{+}-p$ structure.] 
Pour la suite de la discussion, nous introduisons, à côté de la notation électrique $\left(\mathrm{n}, \mathrm{n}^{+}, \mathrm{p}\right)$, une notation structurale : $\mathrm{Si}(\mathrm{a})$ pour le $\mathrm{Si}$ amorphisé, $\mathrm{Si}(\mathrm{c})$ pour le Si monocristallin, et distinguerons :

- l'interface métal-Si(a) à la surface du matériau;

- l'interface $\mathrm{Si}(\mathrm{a})-\mathrm{Si}(\mathrm{c})$ dans la profondeur du matériau.

Les faits suivants :

— insensibilité des propriétés électriques à la nature du métal ;

- variation faible de la hauteur de barrière $\Phi_{\mathrm{B}}$ (vue du côté du métal) en fonction de l'énergie des ions;

- identité du travail de sortie mesuré sur Si-n et Si-p bombardés dans les mêmes conditions [7],

cadrent bien avec la forte différence de concentration en donneurs entre la surface $\left(10^{18} \mathrm{~cm}^{-3}\right)$ et le substrat $\left(10^{15} \mathrm{~cm}^{-3}\right)$ et l'intervention d'un effet tunnel. Il semble probable que la couche de surface amorphe ait des propriétés identiques indépendamment du type $n$ ou $p$ du substrat.

Si donc, en déposant un métal donné sur du Si recouvert d'une couche amorphe dont la nature est indépendante du type de dopage du substrat, on obtient des propriétés radicalement opposées suivant que le substrat est $\mathrm{n}$ ou $\mathrm{p}$, on en déduit que c'est la barrière vue du côté du semiconducteur et donc l'interface $\mathrm{Si}(\mathrm{a})-\mathrm{Si}(\mathrm{c})$ qui détermine les propriétés de transport.

A la limite on peut se demander si la mince couche de surface amorphe fait obstacle au passage du courant. Une telle hypothèse est souvent faite dans le cas de l'interface métal-semiconducteur amorphe $[17,18]$; elle serait à étayer dans le càs du $\mathrm{Si}$ amorphisé par bombardement ionique. Si on supprime l'interface métal-Si(a) (Fig. 8), l'effet de bombardement se résume en un schéma qui rassemble les quatre cas classiques d'une barrière de Schottky [10]. Tout se passe comme si la couche amorphe se comportait comme un métal à faible travail de sortie $\left(\phi_{\mathrm{M}}<\phi_{\mathrm{S}}\right)$.

Il est intéressant de souligner que des jonctions $\mathrm{Si}(\mathrm{a})-\mathrm{Si}(\mathrm{c})$, dans lesquelles le $\mathrm{Si}(\mathrm{a})$ est déposé par évaporation ou par sputtering (tableau II), présentent le même comportement que les jonctions réalisées par bombardement ionique du monocristal : redresseur $\left(\phi_{\mathrm{h}}=0,70-0,75 \mathrm{~V}\right)$ si le substrat est $\mathrm{p}$, ohmique $\left(\Phi_{\mathrm{e}}=0,35-0,45 \mathrm{~V}\right)$ si le substrat est $\mathrm{n}$. Les résultats sont, en première analyse, indépendants du traitement préalable du substrat (attaque chimique ou attaque ionique), de sa résistivité, de la méthode de préparation de la couche amorphe et de son épaisseur (50-3000 $)$.

Cette comparaison renforce l'interprétation selon laquelle c'est l'interface $\mathrm{Si}(\mathrm{a})-\mathrm{Si}(\mathrm{c})$ qui détermine la conduction dans les structures métal-Si(a)-Si(c) préparées par bombardement d'ions argon.

Dans le même ordre d'idées, mentionnons l'effet d'un recuit sur les contacts à base de Si-n bombardé. Sans entrer dans les détails [23], indiquons qu'après bombardement avec des ions argon de $900 \mathrm{eV}$ et recuit à $700{ }^{\circ} \mathrm{C}$, on obtient des caractéristiques analogues à

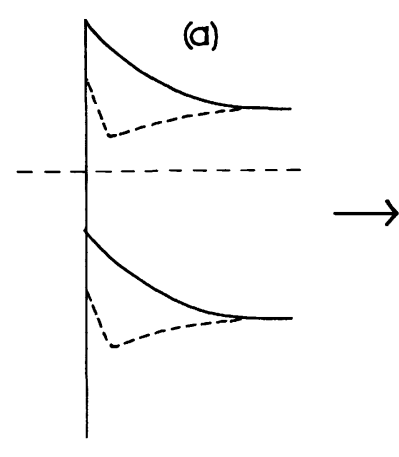

(b)
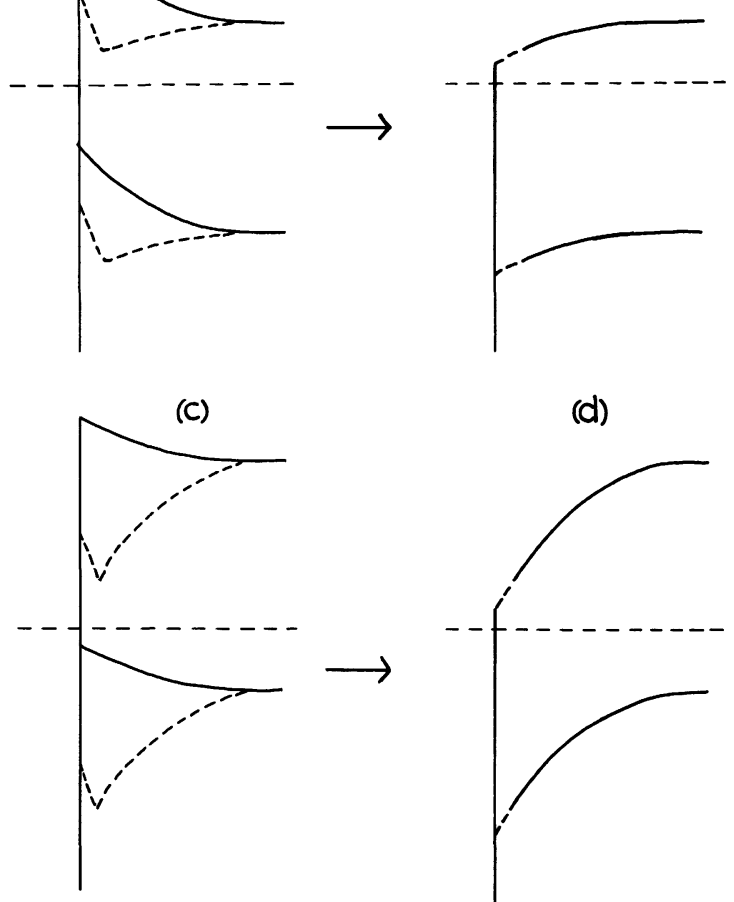

(d)

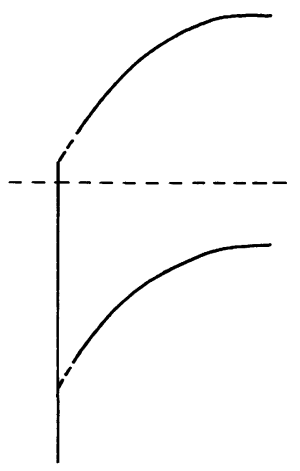

Fig. 8. - Diagrammes de bandes schématisant l'effet d'un bombardement ionique sur du Si-n : a) et du Si-p : c); la courbe en traits pleins correspond à la surface de référence non irradiée, la courbe en pointillés à la surface bombardée. Les schémas b) et d) se déduisent de a) et c) en faisant abstraction de l'interface métal-Si(amorphe).

[Schematic effect of ion bombardment on band diagram : a) Si-n ; b) Si-p; full lines correspond to the undamaged surface, dotted lines to the bombarded surface. Patterns b) and d) result from a) and c) by suppression of the metalamorphous Si interface.]

celles des contacts réalisés par bombardement à faible énergie $(100 \mathrm{eV})$ : caractère passablement redresseur, persistance d'une faible résistance de contact. Or, un recuit à $700^{\circ} \mathrm{C}$ rend la surface du Si monocristalline [5]. La faible résistance de contact est donc indépendante de la nature amorphe ou cristalline de la couche de Si touchant le métal. C'est une preuve supplémentaire de ce que l'interface métal-Si joue un rôle secondaire. Ce n'est plus ici l'interface $\mathrm{Si}(\mathrm{a})-\mathrm{Si}(\mathrm{c})$ qui détermine les propriétés électriques, mais l'interface entre un $\mathrm{Si}$ endommagé et le monocristal, que nous notons : $\mathrm{Si}(\mathrm{d})-\mathrm{Si}(\mathrm{c})$. La persistance de défauts malgré le recuit du Si implanté par des ions argon est un phénomène bien connu : bulles d'argon, micromâcles [24].

Signalons encore l'analogie frappante entre le $\mathrm{Si}$ bombardé par des ions argon et le Si poli mécaniquement. Nous avons testé des contacts réalisés en métallisant $(\mathrm{Ag})$ des échantillons de $\mathrm{Si}$ dont les deux faces sont rodées, la face arrière étant pourvue d'un contact ohmique. La caractéristique de la surface $\mathrm{Si}-\mathrm{n}$ rodée est semblable à celle de la surface Si-n bombardée 


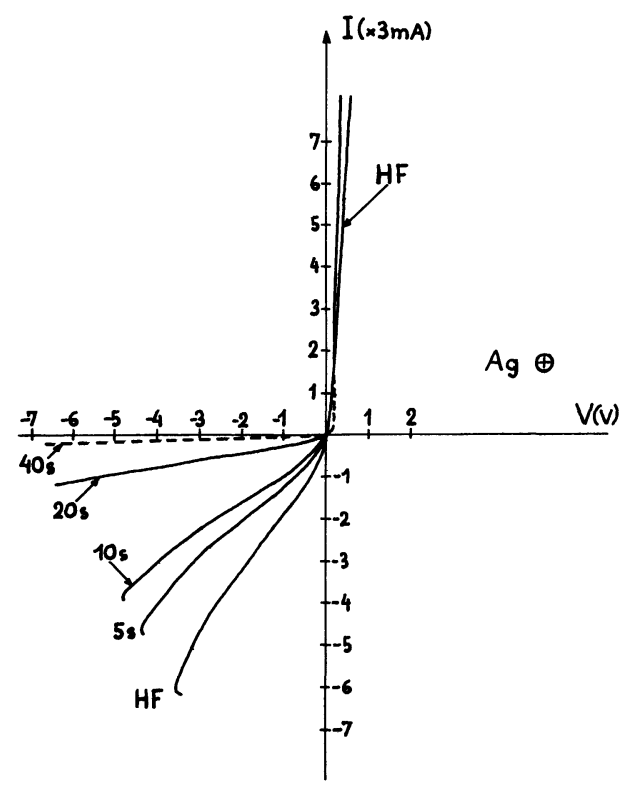

Fig. 9. - Caractéristiques $I(V)$ pour des structures $\mathrm{Ag} / \mathrm{Si}$ $\mathrm{n} / \mathrm{Al}$ à base de $\mathrm{Si}$ rodé, en fonction de la durée de dissolution dans un mélange fluoro-nitrique; la courbe HF correspond à la surface rodée juste débarrassée de la couche d'oxyde natif.

$[I(V)$ curves for $\mathrm{Ag} / \mathrm{Si}-\mathrm{n} / \mathrm{Al}$ structures made with ground $\mathrm{Si}$ as a function of etch time in $\mathrm{HF}+\mathrm{NO}_{3} \mathrm{H}$; the $\mathrm{HF}$ curve is obtained after removal of the native oxide from the ground surface.]

(Fig. 9). En dissolvant par étapes la couche perturbée par le polissage dans un mélange $\mathrm{HF} / \mathrm{CH}_{3} \mathrm{CO}_{2} \mathrm{H} /$ $\mathrm{NO}_{3} \mathrm{H}$, on réalise la même transition qu'en diminuant l'énergie des ions.
Enfin, élargissant encore notre comparaison à d'autres traitements de surface du Si-n, nous relevons qu'un impact de laser produit également de mauvaises diodes [25]. Il s'agit de diodes préparées par métallisation $(\mathrm{Au})$ de Si-n ayant été irradié par une impulsion de laser à rubis. L'augmentation de l'énergie des impulsions provoque une dégradation des caractéristiques $I(V)$ semblable à celle observée pour le bombardement ionique : diminution de $R_{0}$, de $\Phi_{\mathrm{B}}$, augmentation du facteur $n$. Cette dégradation est due à une forte densité de niveaux donneurs (atteignant $6 \times 10^{17} \mathrm{~cm}^{-3}$ à la surface) dont la distribution en fonction de la profondeur peut être approximée par une exponentielle [25]. Le bombardement électronique pulsé (PEBA) entraîne des effets similaires [26].

Un point commun aux traitements de surface ainsi passés en revue est la création d'un interface plus ou moins abrupt entre une couche de surface amorphe (ou fortement désordonnée) et le substrat monocristallin. Cette couche de surface contient une densité élevée de défauts structuraux électriquement actifs, fonctionnant comme donneurs.

Dans une telle structure métal-Si(a ou d)-Si(c), les propriétés de transport apparaissent dominées par l'interface $\mathrm{Si}(\mathrm{a}$ ou d)-Si(c). Le contact métal-Si joue un rôle mineur sauf pour les faibles perturbations où il tend vers une barrière de Schottky classique.

\section{Remerciements.}

Nous tenons à remercier tout particulièrement M. J. P. Ponpon (CRN, Strasbourg) pour ses critiques et nombreuses suggestions.

Bibliographie

[1] Carrière, B. et Deville, J. P., Surface Sci. 80 (1979) 278.

[2] Lang, B., Scholler, P. et Carrière, B., Surface Sci. 99 (1980) 103.

[3] LANG, B. et Mosser, A., J. Microsc. Spectrosc. Electron. 6 (1981) 131.

[4] Carrière, B., Chouiyakh et Lang, B., Surface Sci. 126 (1983) 495.

[5] Cheheb, A., Thèse 3e cycle, Strasbourg (1983).

[6] Fonash, S. J., Ashok, S. and Singh, R., Appl. Phys. Lett. 39 (1981) 423.

[7] Testemale, E., Palau, J. M., Ismaïl et Lassabatère, L., Solid State Electron. 26 (1983) 325.

[8] Lau, S. S.. J. Vacuum Sci. Technol. 15 (1978) 1656.

[9] Chang, C. Y., Fang, Y. K. and Sze, S. M., Solid State Electron. 14 (1971) 541.

[10] RHODERICK, E. H., Metal-Semiconductors Contacts, ed. P. Hammond and D. Walsh (Clarendon Press, Oxford) 1980.

[11] Popovic, R. S., Solid State Electron. 21 (1978) 1133.

[12] Law, J. T., J. Phys. Chem. Solids 14 (1960) 9.

[13] Heiland, G. and Lamatsch, H., Surface Sci. 2 (1964) 18.
[14] PaI, Y. P. and Lin, H. C., Solid State Electron. 24 (1981) 929.

[15] Williams, R. S., Solid State Commun. 41 (1982) 153.

[16] Müller, G. and Kalbitzer, S., Philos. Mag. B41 (1980) 307.

[17] Sharma, B. L., Semicond. Semimet. 15 (1981) 1.

[18] Wey, H., Phys. Rev. B 13 (1976) 3495.

[19] JAYADEVAiah, T. S. and BusmundRud, O., Electron. Lett. 8 (1972) 75.

[20] Ali, M. P., Tove, P. A. and Norde, H., Phys. Scripta 24 (1981) 399.

[21] MeI, L. and Greene, J. E., J. Vacuum Sci. Technol. 11 (1974) 145.

[22] Koshy, J., Phys. Status Solidi, A 79 (1983) K21.

[23] ChouiYaKh, A. et LANG, B. (en préparation).

[24] Revesz, P., Wittmer, M., Roth, J. and MAYer, J. W., J. Appl. Phys. 49 (1978) 5199.

[25] Ponpon, J. P., Buttung, E. et Siffert, P., Rev. Phys. Appl. 17 (1982) 687.

[26] Doghmane, M. S., Barbier, D. et Laugier, A., J. Physique Colloq. 44 (1983) C5-297. 